\title{
Confidence Sets for the Aumann Mean of a Random Closed Set
}

\author{
Raffaello Seri ${ }^{1}$ and Christine Choirat $^{2}$ \\ 1 Università degli Studi dell'Insubria, 21100 Varese, Italy, \\ raffaello.seri@uninsubria.it \\ 2 Università degli Studi dell'Insubria, 21100 Varese, Italy, \\ christine.choirat@uninsubria.it
}

\begin{abstract}
The objective of this paper is to develop a set of reliable methods to build confidence sets for the Aumann mean of a random closed set estimated through the Minkowski empirical mean. In order to do so, we introduce a procedure to build a confidence set based on Weil's result for the Hausdorff distance between the empirical and the Aumann means; then, we introduce another procedure based on the support function.
\end{abstract}

\section{Introduction}

In this paper we consider algorithms for deriving confidence regions for the mean of a sample of observed objects and shapes represented as closed and bounded (i.e. compact) sets in the Euclidean space $\mathbb{R}^{d}$. In order to obtain these results we rely on the powerful theory of Random Closed Sets. We suppose to observe a sample of $n$ independent identically distributed realizations of a random element, say $\mathfrak{X}$, taking on its own values in the class of compact sets of $\mathbb{R}^{d}$. A precise definition of confidence region will be given in the following, but, in the meanwhile, the reader should interpret it as a region of the space containing $\mathbb{E} \mathfrak{X}$ with prescribed probability (e.g. $95 \%$ ) on the basis of our sample.

Almost the same technique can be used to obtain a confidence region for a set observed with error. In both cases, the sets need not be completely observed (it is indeed enough to observe their support lines on a grid of directions); this allows, as will be discussed in the following, for applying the technique to observations derived through computerized tomography (see Natterer, 1986, Kak and Slaney, 1988, Gardner, 1995), tactile sensing and laser-radar systems.

In tactile sensing, a robot jaw composed of two parallel plates is clamped onto an object, thus measuring its extension in the direction perpendicular to the plates. In the 2-dimensional case, if the jaw is perpendicular with respect to the plane of the object, the lines corresponding to the plates are called "support lines". When the jaw moves with respect to the object, the support lines describe a polygonal approximation of the set. If the set is convex, this approximation can be made as precise as needed.

In laser-radar systems (or LIDAR, LIght Detection And Ranging), a laser beam is sent towards the object. The part of the beam that is reflected allows 
for measuring the distance between the source of the radiation and the "grazing plane" (that is, the plane perpendicular to the direction of the beam and tangential to the object). In LIDAR range-and-orientation measurement, the position of the laser can vary, thus allowing for constructing a collection of planes (the grazing planes, indeed) circumscribing the shape of interest.

In computerized tomography, the support lines of the object are recorded as a subproduct of the calculation of the absorption density of the body. However, the important fact is that, in this case as well, it is possible, for a specified direction, to identify the planes passing through the extreme points of the object.

All of these applications are mostly restricted to objects in 2-dimensional spaces: our technique though is more general since it can be used for higher dimensional Euclidean spaces (even if some limitations due to a curse of dimensionality phenomenon suggest prudent applications to problems with $d \geq 4$ ).

\section{Some Results on Random Sets}

After the first pioneering works (see Kendall, 1974, Matheron, 1975), the study of random sets has been receiving growing attention in the literature (see Goutsias, 1997, Molchanov, 1997): random sets have proved to be a valuable modelling tool in Economics, Physics and Biology and their theory offers a suitable framework to analyze old problems (e.g., epigraphical convergence in statistical estimation, see Hess, 1996, Choirat et al., 2003). The only feasible way to compare random sets (see e.g. Reyment, 1982, Stoyan and Stoyan, 1994) consists in identifying the shape of the random set with some of its measurements (length, perimeter, area, etc.) and to calculate them on a sample: clearly the choice of the measurements underlying this procedure relies heavily on the statistician's experience.

To overcome these difficulties (i.e. relying on some arbitrary shape measurements), we consider the mean of the random objects. The most convenient choice is the so-called Aumann mean since Central Limit Theorems for this case have already been derived. First, we need to introduce a certain number of preliminary concepts that will be used in the following.

The distance function from a point $x$ to the set $C \subset \mathbb{R}^{d}$ is:

$$
d(x, C) \triangleq \inf _{y \in C} d(x, y)
$$

The support function of $C$ is:

$$
\mathrm{h}(y, C)=\mathrm{h}_{C}(y) \triangleq \sup _{x \in C}\langle y, x\rangle=\sup _{x \in C}\left(\sum_{i=1}^{d} y_{i} x_{i}\right)
$$

where $y \in \mathbb{S}^{d-1} \triangleq\left\{u \in \mathbb{R}^{d}:\|u\|=1\right\}$; it characterizes completely a closed convex set. The support function of a set $C \subset \mathbb{R}^{d}$ is an element of $\mathcal{C}\left(\mathbb{S}^{d-1}\right)$, the collection of continuous functions defined on the unit sphere $\mathbb{S}^{d-1}$.

The Hausdorff distance between two sets $C$ and $C^{\prime}$ is defined by

$$
\rho_{H}\left(C, C^{\prime}\right) \triangleq \max \left\{\sup _{x \in C} d\left(x, C^{\prime}\right), \sup _{x^{\prime} \in C^{\prime}} d\left(x^{\prime}, C\right)\right\} .
$$


The norm of a set $C$ is simply:

$$
\|C\|=\rho_{H}(C,\{0\}) .
$$

The Minkowski sum of two sets $A$ and $B$ is defined by:

$$
A \oplus B \triangleq\{x+y: x \in A, y \in B\} ;
$$

in the following, we will set:

$$
\bar{X}_{n} \triangleq \frac{1}{n} \bigoplus_{i=1}^{n} X_{i}
$$

The scalar multiplication is defined as:

$$
\alpha C \triangleq\{\alpha x: x \in C\} .
$$

We denote by $\mathfrak{C}$ the set of all nonempty closed subsets of $\mathbb{R}^{d}$. Consider a setvalued map (alias multifunction, correspondence) $\mathfrak{X}$ from the probability space $(\Omega, \mathcal{A}, \mathbb{P})$ to $\mathfrak{C}$. A map $\mathfrak{X}$ from $\Omega$ into $\mathfrak{C}$ is said to be $\mathcal{A}$-measurable if for every open subset $U$ of $\mathbb{R}^{d}$, the set $\{\omega \in \Omega: \mathfrak{X}(\omega) \cap U \neq \varnothing\}$ is a member of $\mathcal{A}$. A measurable set-valued map is also called a random closed set, or RACS for short.

The Aumann mean of a RACS can be characterized through the support function as the set $\mathbb{E} \mathfrak{X}$ such that the following equality holds: ${ }^{3}$

$$
\mathrm{h}(\cdot, \mathbb{E} \mathfrak{X}) \triangleq \mathbb{E} h(\cdot, \mathfrak{X}) .
$$

A well known result (see Artstein and Vitale, 1975) states that RACS satisfy a Law of Large Numbers.

Theorem 1. Let $X_{1}, X_{2}, \ldots$ be a sequence of iid random sets in $\mathbb{R}^{d}$ with $\mathbb{E}\|\mathfrak{X}\|<$ $\infty$. Then

$$
\bar{X}_{n} \stackrel{\text { as }}{\longrightarrow} \mathbb{E} \mathfrak{X} \text {. }
$$

The Aumann mean is always a convex set, but, even if the random set $\mathfrak{X}$ is not convex-valued, the Shapley-Folkman's inequality implies that the Hausdorff distance between $\frac{1}{n} \bigoplus_{i=1}^{n} X_{i}$ and of $\frac{1}{n} \bigoplus_{i=1}^{n} \operatorname{co} X_{i}$ (where co $X_{i}$ is the convex hull of $X_{i}$ ) goes to 0 for large $n$.

A CLT for RACS can be obtained applying the CLT for $\mathcal{C}\left(\mathbb{S}^{d-1}\right)$-valued random variables (see Araujo and Giné, 1980) to the support functions.

Theorem 2. Let $X_{1}, X_{2}, \ldots$ be a sequence of iid random sets in $\mathbb{R}^{d}$ with $\mathbb{E}\|\mathfrak{X}\|^{2}<$ $\infty$. Then

$$
\sqrt{n} \cdot\left(\mathrm{h}(\cdot, \mathbb{E} \mathfrak{X})-\mathrm{h}\left(\cdot, \bar{X}_{n}\right)\right) \stackrel{\mathcal{D}}{\longrightarrow} Z(\cdot),
$$

where $Z$ is a Gaussian centered process on $\mathbb{S}^{d-1}$ of covariance function $\Gamma_{\mathfrak{X}}(u, v) \triangleq$ $\mathbb{E} Z(u) Z(v)$.

\footnotetext{
3 This characterization holds only when the probability space is non-atomic, which is obviously the case here. See Artstein and Vitale (1975) for more details.
} 
A fundamental result is Hörmander's formula. It relates the Hausdorff distance between sets to the $L_{\infty}$-distance between support functions:

$$
\rho_{H}\left(C, C^{\prime}\right)=\sup _{y \in \mathrm{B}}\left|\mathrm{h}(y, C)-\mathrm{h}\left(y, C^{\prime}\right)\right|
$$

From this result, Weil obtains the following limit theorem for the Hausdorff distance between the empirical Minkowski mean of a sample of iid RACS and its Aumann mean.

Corollary 1. Let $X_{1}, X_{2}, \ldots$ be a sequence of iid random sets in $\mathbb{R}^{d}$ with $\mathbb{E}\|\mathfrak{X}\|^{2}<$ $\infty$. Then

$$
\sqrt{n} \cdot \rho_{H}\left(\bar{X}_{n}, \mathbb{E} \mathfrak{X}\right) \stackrel{\mathcal{D}}{\longrightarrow} \sup _{u \in \mathbb{S}^{d-1}}|Z(u)|
$$

where $Z$ is a Gaussian centered process on $\mathbb{S}^{d-1}$ of covariance function $\Gamma_{\mathfrak{X}}(u, v)=$ $\mathbb{E} Z(u) Z(v)$.

A quick glance shows that Weil's result, stated in terms of the Hausdorff distance, is weaker than the one of Theorem 2. As already mentioned, we will develop two procedures for building confidence sets, one based on Weil's Theorem and the other on the original result on the support function. Therefore, Section 2 deals with Weil's type confidence sets and Section 3 with support function confidence sets. Section 4 presents an application to simulated data and Section 5 briefly summarizes some future developments that will be presented in a companion paper.

\section{Weil's Type Confidence Sets}

In the following, we suppose that the asymptotic approximation suggested by Corollary 1 is also valid for finite $n$; therefore, we start from equation

$$
1-\alpha=\mathbb{P}\left\{\sqrt{n} \cdot \rho_{H}\left(\frac{1}{n} \bigoplus_{i=1}^{n} \operatorname{co} X_{i}, \mathbb{E} \mathfrak{X}\right) \leq \gamma\right\}
$$

remark that this is not in the form of a confidence set, since it is not possible to write it as:

$$
1-\alpha \leq \mathbb{P}\left\{\mathbb{E} \mathfrak{X} \subset C_{\alpha}\left(X_{i}, i=1, \ldots, n\right)\right\}
$$

where $C_{\alpha}\left(X_{i}, i=1, \ldots, n\right)$ is the confidence set based on the sample $\left(X_{i}\right)_{i=1, \ldots, n}$. However, we can write it as: ${ }^{4}$

${ }^{4}$ In the derivation, we use the characterization of the Hausdorff distance as:

$$
\rho_{H}\left(C, C^{\prime}\right)=\inf \left\{\alpha: C \subseteq C^{\prime} \oplus \alpha \mathrm{B} \text { and } C^{\prime} \subseteq C \oplus \alpha \mathrm{B}\right\}
$$

where B is the closed unit ball. 


$$
\begin{aligned}
1-\alpha & =\mathbb{P}\left\{\rho_{H}\left(\frac{1}{n} \bigoplus_{i=1}^{n} \operatorname{co} X_{i}, \mathbb{E} \mathfrak{X}\right) \leq \frac{\gamma}{\sqrt{n}}\right\} \\
& =\mathbb{P}\left\{\operatorname { i n f } \left[\beta>0:\left(\frac{1}{n} \bigoplus_{i=1}^{n} \operatorname{co} X_{i}\right) \subseteq \mathbb{E} \mathfrak{X} \oplus \beta \mathrm{B}\right.\right. \\
& \text { and } \left.\left.\mathbb{E} \mathfrak{X} \subseteq\left(\frac{1}{n} \bigoplus_{i=1}^{n} \operatorname{co} X_{i}\right) \oplus \beta \mathrm{B}\right] \leq \frac{\gamma}{\sqrt{n}}\right\} \\
& \leq \mathbb{P}\left\{\left(\frac{1}{n} \bigoplus_{i=1}^{n} \operatorname{co} X_{i}\right) \subseteq \mathbb{E} \mathfrak{X} \oplus \frac{\gamma}{\sqrt{n}} \mathrm{~B} \text { and } \mathbb{E} \mathfrak{X} \subseteq\left(\frac{1}{n} \bigoplus_{i=1}^{n} \operatorname{co} X_{i}\right) \oplus \frac{\gamma}{\sqrt{n}} \mathrm{~B}\right\} \\
& \leq \mathbb{P}\left\{\mathbb{E} \mathfrak{X} \subseteq\left(\frac{1}{n} \bigoplus_{i=1}^{n} \operatorname{co} X_{i}\right) \oplus \frac{\gamma}{\sqrt{n}} \mathrm{~B}\right\} .
\end{aligned}
$$

Remark that this confidence set is not exact in general, that is the inequality cannot be substituted by an equality sign, not even asymptotically.

Our aim is to find an approximate value of $\gamma$ from (1) and to put it in (2).

From Weil's Theorem, we have:

$$
\begin{aligned}
& \sqrt{n} \cdot \rho_{H}\left(\frac{1}{n} \bigoplus_{i=1}^{n} \operatorname{co} X_{i}, \mathbb{E} \mathfrak{X}\right)=\sqrt{n} \cdot \sup _{u \in \mathbb{S}^{d-1}}\left|\frac{1}{n} \sum_{i=1}^{n} \frac{\mathrm{h}\left(u, X_{i}\right)}{n}-\mathbb{E} \mathrm{h}(u, \mathfrak{X})\right| \\
& \underset{n \rightarrow \infty}{\stackrel{\mathcal{D}}{\longrightarrow}} \sup _{u \in \mathbb{S}^{d-1}}|Z(u)|,
\end{aligned}
$$

where $Z$ is a centered random variable of $\mathcal{C}\left(\mathbb{S}^{d-1}\right)$. Therefore, for $n \rightarrow \infty$, we have:

$$
1-\alpha=\mathbb{P}\left\{\sup _{u \in \mathbb{S}^{d-1}}|Z(u)| \leq \gamma\right\}=\mathbb{P}\left\{|Z|^{\max } \leq \gamma\right\},
$$

where we set $|Z|^{\max } \triangleq \sup _{u \in \mathbb{S}^{d-1}}|Z(u)|$.

Since the distribution of $|Z|^{\max }$ is unknown, we introduce two approximations of this formula in order to obtain a confidence set:

1. we approximate the distribution of $|Z|^{\max }$ through the distribution of $|Z|_{p}^{\max }$, defined by:

$$
|Z|_{p}^{\max } \triangleq \max _{i=1, \ldots, p}\left|Z\left(u_{i}\right)\right|, \quad u_{i} \in \mathbb{S}^{d-1},
$$

that is the $p$-points approximation of $|Z|^{\max }$;

2. if we set

$$
\mathbf{Z} \triangleq\left[\begin{array}{c}
Z\left(u_{1}\right) \\
\ldots \\
Z\left(u_{p}\right)
\end{array}\right],
$$

we have:

$$
\mathbf{Z} \sim \mathcal{N}[\mathbf{0}, \mathbb{V}(\mathbf{Z})]
$$


or equivalently

$$
\begin{gathered}
\zeta=\mathbb{V}(\mathbf{Z})^{-\frac{1}{2}} \mathbf{Z} \sim \mathcal{N}[\mathbf{0}, \mathbf{I}], \\
\mathbf{Z}=\mathbb{V}(\mathbf{Z})^{\frac{1}{2}} \zeta \sim \mathcal{N}[\mathbf{0}, \mathbb{V}(\mathbf{Z})]
\end{gathered}
$$

therefore, for $|Z|_{p}^{\max }$, (1) becomes:

$$
\begin{aligned}
1-\alpha & =\mathbb{P}\left\{\max _{i=1, \ldots, p}\left|Z\left(u_{i}\right)\right| \leq \gamma_{p}\right\} \\
& =\mathbb{P}\left\{\max _{i=1, \ldots, p}|\mathbf{Z}| \leq \gamma_{p}\right\} \\
& =\mathbb{P}\left\{\max _{i=1, \ldots, p}\left|\mathbb{V}(\mathbf{Z})^{\frac{1}{2}} \zeta\right| \leq \gamma_{p}\right\}
\end{aligned}
$$

unfortunately, $\mathbb{V}(\mathbf{Z})$ is not known a priori and it has to be estimated through a consistent estimator, say $\widehat{\mathbb{V}(\mathbf{Z})}$, to get

$$
\widehat{\mathbb{V}(\mathbf{Z})}^{\frac{1}{2}} \zeta=\widehat{\mathbf{Z}}
$$

and

$$
1-\alpha=\mathbb{P}\left\{\max _{i=1, \ldots, p}|\mathbf{Z}| \leq \gamma_{p}\right\}=\mathbb{P}\left\{\max _{i=1, \ldots, p}|\widehat{\mathbf{Z}}| \leq \gamma_{p n}\right\}
$$

\section{Support Function Confidence Sets}

Alternatively, we could obtain a confidence set for the average support function $\mathrm{h}(\cdot, \mathbb{E} \mathfrak{X})$ as the set of all the centered support functions that are upper bounded by a constant $\lambda$ :

$$
\begin{aligned}
1-\alpha & =\mathbb{P}\left\{\sqrt{n}\left(\mathrm{~h}(u, \mathbb{E} \mathfrak{X})-\mathrm{h}\left(u, \bar{X}_{n}\right)\right) \leq \lambda, \forall u \in \mathbb{S}^{d-1}\right\} \\
= & \mathbb{P}\left\{\sup _{u \in \mathbb{S}^{d-1}}\left[\sqrt{n}\left(\mathrm{~h}(u, \mathbb{E} \mathfrak{X})-\mathrm{h}\left(u, \bar{X}_{n}\right)\right)\right] \leq \lambda\right\} .
\end{aligned}
$$

Therefore:

$$
\sup _{u \in \mathbb{S}^{d-1}}\left[\sqrt{n}\left(\mathrm{~h}(u, \mathbb{E} \mathfrak{X})-\mathrm{h}\left(u, \bar{X}_{n}\right)\right)\right] \stackrel{\mathcal{D}}{\longrightarrow} \sup _{u \in \mathbb{S}^{d-1}} Z(u),
$$

and we set

$$
Z^{\max } \triangleq \sup _{u \in \mathbb{S}^{d-1}} Z(u)
$$

We use the same approximation strategy as before:

1. since the distribution of the random variable $Z^{\max }$ is not known, we approximate it through the random variable $Z_{p}^{\max }$, defined as:

$$
Z_{p}^{\max } \triangleq \max _{i=1, \ldots, p} Z\left(u_{i}\right), \quad u_{i} \in \mathbb{S}^{d-1}
$$


2. as before, we set

$$
\widehat{\mathbb{V}(\mathbf{Z})}^{\frac{1}{2}} \zeta=\widehat{\mathbf{Z}}
$$

and

$$
1-\alpha=\mathbb{P}\left\{\max _{i=1, \ldots, p} \mathbf{Z} \leq \lambda_{p}\right\}=\mathbb{P}\left\{\max _{i=1, \ldots, p} \widehat{\mathbf{Z}} \leq \lambda_{p n}\right\}
$$

\section{A Simulation Study}

In order to present the techniques developed in the previous Sections, we analyze some simulated data. ${ }^{5}$

The following simulated data are generated as the convex hull of 5 points drawn from two independent standard normal variates. Figures 1 and 2 are drawn for a discretization with $p=10$ and a sample of size $n=50$. The dimension of the sample has the same order of magnitude than real data.

For any set $X_{i}, i=1, \ldots, n$, a $p$-points discretized version of the support function $\mathrm{h}\left(x_{j}, X_{i}\right), j=1, \ldots, p$, is drawn. The mean and the variance of these functions are calculated and are used to derive, in Figure 1, the empirical cumulative distribution function of $|\widehat{Z}|_{p}^{\max }$ (Weil) and $\widehat{Z}_{p}^{\max }$ (Supp). Since their evaluation requires the integration of the density of a high-dimensional normal random vector over a rectangular domain, we have used a simulated procedure, the GHK simulator (Geweke-Hajivassiliou-Keane, see e.g. Hajivassiliou et al., 1996), with $s=1000$ simulations in order to approximate the integral.

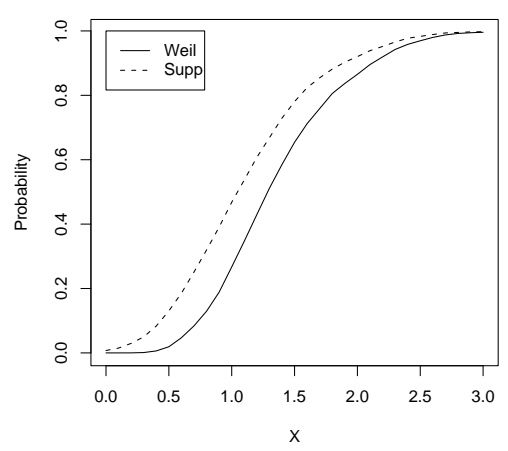

Fig. 1. The cumulative distribution function of $\widehat{Z}_{p}^{\max }$ (Weil) and $\widehat{Z}_{p}^{\max }$ (Supp)

\footnotetext{
${ }^{5}$ The following simulations have been programmed in $\mathrm{R}$, a free software that can be downloaded from http://www.r-project.org. See Ihaka and Gentleman (1996).
} 
From these two distribution functions the values of $\gamma_{p n}$ and $\lambda_{p n}$ satisfying:

$$
\begin{aligned}
& 1-\alpha=\mathbb{P}\left\{|\widehat{Z}|_{p}^{\max } \leq \gamma_{p n}\right\} \\
& 1-\alpha=\mathbb{P}\left\{\widehat{Z}_{p}^{\max } \leq \lambda_{p n}\right\}
\end{aligned}
$$

are obtained through an iterative procedure. In this case too, the distributions of $|\widehat{Z}|_{p}^{\max }$ and $\widehat{Z}_{p}^{\max }$ have been approximated through the GHK simulator, with $s=30$ simulations. These values are then used in order to obtain confidence sets as described in Sections 2 and 3. In Figure 2, Mean is the Minkowski mean of the sample, Weil is the confidence set described in Section 2 and Supp is the confidence set of Section 3. It is evident, even from this example, that the procedure based on the support function yields smaller confidence set than the one based on Weil's Theorem 1.

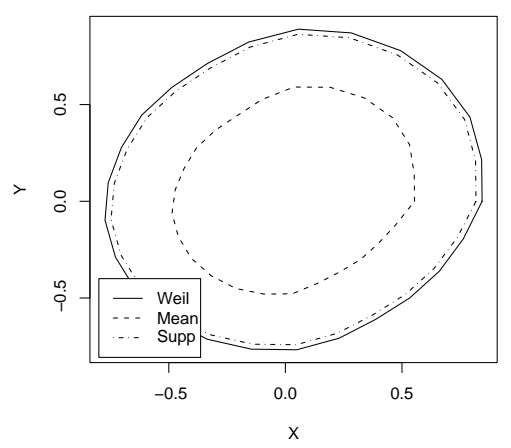

Fig. 2. Minkowski mean (Mean) of a sample and confidence sets (Weil, Supp) for the Aumann mean

Clearly, a simple inspection of the formulas should convince the reader that the values of $\gamma_{p n}$ and $\lambda_{p n}$ are expected to increase with $p$. Table 1 shows the values of $\gamma_{p n}$ and $\lambda_{p n}$ for different $p$ and $n$, where the number of simulation $s$ is fixed to 30 and the number of replications to 200 .

Figure 3 shows the kernel estimators based on a bandwidth of 0.15 of the densities of $\gamma_{p n}$ and $\lambda_{p n}$ for $p=12$ and $n=50,30$ simulations and 200 replications. It is evident from the data that the confidence set based on the support function is smaller than the one based on the Hausdorff distance. 
Table 1. $\gamma_{p n}$ and $\lambda_{p n}$ for confidence sets (standard errors in parentheses)

\begin{tabular}{|c|c|c|c|}
\hline$(\mathbf{p}, \mathbf{n})$ & $\mathbf{2 5}$ & $\mathbf{5 0}$ & $\mathbf{1 0 0}$ \\
\hline \multicolumn{4}{|c|}{$\gamma_{p n}$} \\
\hline 10 & 2.244608 & 2.253287 & 2.230253 \\
& $(0.2498404)$ & $(0.2095767)$ & $(0.1877489)$ \\
\hline 30 & & & 2.23936 \\
& & $\lambda_{p n}$ & $(0.2307905)$ \\
\hline \multicolumn{4}{|c|}{} \\
\hline 10 & 2.041577 & 2.042199 & 2.031207 \\
& $(0.2600087)$ & $(0.2019208)$ & $(0.1811784)$ \\
\hline 30 & & & 2.045988 \\
& & $0.2263206)$ \\
\hline
\end{tabular}

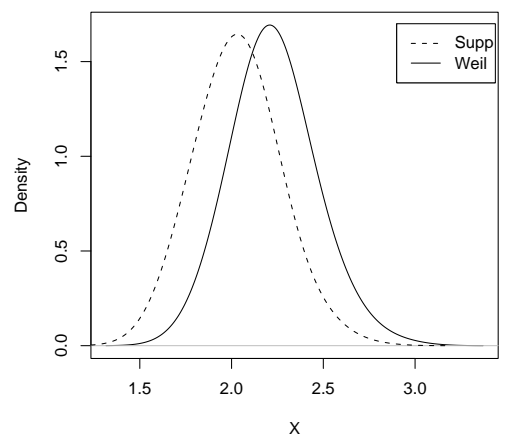

Fig. 3. Kernel estimators of the density of $\lambda_{p n}$ (Supp) and $\gamma_{p n}$ (Weil)

\section{Further Developments}

A companion paper (see Choirat and Seri, 2003) shows that the previous procedures, for $p, n \rightarrow \infty$, yield consistent confidence sets for the Aumann mean of a RACS and establishes their rates of convergence: moreover, the confidence set based on the support function is shown to dominate strictly the one based on the Hausdorff distance. At last, a limited simulation study illustrates the feasibility and the precision of the present approaches.

\section{References}

Araujo A., Giné E.: The central limit theorem for real and Banach valued random variables, Wiley, New York (1980)

Artstein Z., Vitale R.A.: A strong law of large numbers for random compact sets. The Annals of Probability 3 (1975) 879-882 
Choirat C., Seri R.: Confidence sets for the Aumann mean of random closed sets. Working Paper, Université Paris 9 Dauphine (2003)

Choirat C. ,Hess C., Seri R.: A Functional Version of the Birkhoff Ergodic Theorem for a Normal Integrand: A Variational Approach. The Annals of Probability 31 (2003) 63-92

Gardner R.J.: Geometric tomography, Encyclopedia of mathematics and its applications 58 Cambridge University Press (1995)

Goutsias J.: Morphological analysis of random sets, an introduction. In: Random sets, theory and applications, J. Goutsias, R.P. Mahlher, H.T. Nguyen. eds, Springer (1997) $2-26$

Hajivassiliou V., McFadden D.L., Ruud P.: Simulation of multivariate normal rectangle probabilities and their derivatives: Theoretical and computational results. Journal of Econometrics 72 (1996) 85-134

Hess C.: Epi-convergence of sequences of normal integrands and strong consistency of the maximum likelihood estimator. The Annals of Statistics 24 (1996) 1298-1315

Ihaka R., Gentleman R.: R: a language for data analysis and graphics. Journal of Computational and Graphical Statistics 5 (1996) 299-314

Kak A.C., Slaney M.: Principles of computerized tomographic imaging, IEEE Press (1988)

Kendall .G.: Foundations of a theory of random sets. In: Advances in theory and applications of random sets, E.F. Harding, D.G. Kendall eds, Wiley, London (1974) 322-376

Matheron G.: Random sets and integral geometry, Wiley, New York (1975)

Molchanov I.S.: Statistical models for random sets. In: Random sets, theory and applications, J. Goutsias, R.P. Mahlher, H.T. Nguyen eds, Springer (1997) 27-45

Natterer F.: The mathematics of computerized tomography, Wiley, Stuttgart (1986)

Reyment R.A.: Multivariate Morphometrics. In: Handbook of Statistics, Volume 2, P.R. Krishnaiah and L.N. Kanal eds, North-Holland Publishing Company (1982) 721-745

Stoyan D., Stoyan H.: Fractals, random shapes and point fields, Wiley, Chichester (1994)

Weil W.: An application of the central limit theorem for Banach-space-valued random variables to the theory of random sets. Zeitschrift für Wahrscheinlichkeitstheorie und verwandte Gebiete 60 (1982) 203-208 